\title{
Code 6: Penile Shrinkage and Patient Expectations of Side Effects
}

Patients discovered they had side effects after the operation, that they were not expected (Table 51.1). This included penile shrinkage when the prostate was removed.

- 'There was a decrease in penile length.'-ID 4

- 'We need therapy for ED- this impacts on our body image.'-ID1

They felt management of patient expectations for this needed to be better preoperatively, again, supporting the use of a pathway or pathway pre-surgery. As part of the pathway, they requested side effects of surgery, pre-operative counselling and psychosexual care to be discussed preoperatively.

Table 51.1 Code 6: Penile shrinkage and being warned about side effects

\begin{tabular}{l|l}
\hline & Patient identification using Patient Identification Numbers \\
\hline Side effects of surgery & 'There was penile shrinkage afterwards.'-ID 3 \\
\hline $\begin{array}{l}\text { Requirement for } \\
\text { pathway }\end{array}$ & 'We need something to address these issues.'-ID 4 \\
\hline Timing of pathway & $\begin{array}{l}\text { 'Any intervention for ED needs to come after incontinence } \\
\text { settles.'-ID5 }\end{array}$ \\
\hline
\end{tabular}

